\title{
Fuzzy Inference System Based on a Model of Affective- Cognitive Criteria for English Learning Achievement
}

\author{
Fitra A. Bachtiar* , Gunadi H. Sulistyo ${ }^{\dagger}$, \\ Eric W. Cooper ${ }^{\ddagger}$, and Katsuari Kamei ${ }^{\ddagger}$
}

\begin{abstract}
Criterion-referenced assessment (CRA) employs a specifically-defined set of criteria or standards that can guide teachers to assess students grade by comparing students' learning score with the pre-specified standards. However, the use of CRA is considered incomplete as most of the criteria are merely based on knowledge domains. Meanwhile, affective factors also need to be considered in the assessment to describe students' complete attributes. Nonetheless, measuring affective factors is not as straightforward task as measuring cognitive factors because affective descriptions is often represented in descriptive verbal terms. In this study, affective factors and cognitive factors based on CRA are combined as a model for assessment of students' learning. A questionnaire is developed to collect student affective attributes. A novel fuzzy inference system (FIS) is proposed to infer student achievement in English learning based on CRA. The FIS method was applied to analyze the data collected from students studying English as a second language. The result indicates the usefulness of the FIS based on CRA as a basis to assess student English learning by considering both affective and cognitive factors.
\end{abstract}

Keywords: affective, cognitive, fuzzy, inference, fuzzy membership, fuzzy rules

\section{Introduction}

Assessment in the context of learning is used to establish valid and reliable evaluation of student learning outcomes in the form of scores that reflects student learning achievement [1]. The outcomes of the assessment are an appraisal of student achievement that has to meet learning expectations [2]. The process of assessment includes systematic gathering, synthesizing, evaluating, and interpreting evidence to determine of how well students are able to meet learning objectives of a subject in a sufficient attempts [3].

The assessment process in general involves assigning tasks with corresponding weights to label the significance of the relative tasks. To get the final result of the assessment, the score obtained from each assessment task is added and projected in a linear approach

\footnotetext{
* Graduate School of Information Science and Engineering, Ritsumeikan University, Kusatsu, Shiga, Japan.

$\dagger$ English Department, Faculty of Letters, State University of Malang, Malang, East Java, Indonesia.

* College of Information Science and Engineering, Ritsumeikan University, Kusatsu, Shiga, Japan.
} 
[4]. This method is considered intuitive since the scoring is commonly not based on an empirically-defined set of skills. Another type of assessment that requires a criterion to measure student achievement, known as criterion-referenced assessment (CRA), has been reported by several researchers [2][3][5]. The criteria, a set of clearly defined learning objectives, are used by the teacher as a guidance to determine the student grade [3][4]. CRA is a more recent approach to assessment [6]. However, most of developed criteria used to evaluate students are concerned only with student knowledge.

Affective factors have attracted a number of researchers' attention in recent years. Conceptually, there is a connection of affect, cognition, and social functioning, the critical role of emotion in education [7], and the need to include emotion in learning [8]. Anderson et al. [9] reiterated Bloom's ideas that three domains need considering in teaching and learning, namely cognitive, affective, and psychomotor domain. The affective domain is associated with student emotional attributes, for example motivation and attitude. Measuring cognitive factors can be considered as a straightforward task as this activity can be carried out by checking student understanding accurately of a topic by giving a score. However, quantifying affective factors cannot be performed in a similar fashion to quantifying cognitive factors. Teachers normally state student affective factors with verbal terms, for example, "I see student A is very anxious when I tried to ask him a question", rather than quantify affective factors with numbers.

This paper proposes a FIS-based CRA model using scoring criteria based on student affective and cognitive factors. The assessment method is an alternative to the standard grading that involves only student cognitive performances. Similar systems have been concerned only with cognitive domain assessment and have not attempted to assess student cognitive and affective criteria. This studies also identifies affective factors that influence student in English language learning.

\section{Related Works}

Researchers have presented methods of student assessment using fuzzy approaches. Studies in investigating student assessment using fuzzy sets can be seen in an earlier work by Biswas [10]. In that study, an evaluation of student answer scripts based on fuzzy sets is introduced. The answer script evaluation is proposed by generalizing a method which uses matrix-valued marking. Chen and Lee [11] proposed a method that extends Biswas' work to include the matching operations and fairness in evaluating the answer script. Bai and Chen [12] further expand the earlier work of grading systems using fuzzy membership functions and fuzzy rules. In their work, difficulty, importance, and complexity of questions for student answer script evaluation are considered.

In the field of language learning, Cin and Baba [2] proposed multi-criteria assessment to evaluate students. Student English performance is determined by the total result of the assessment of the criteria, based on different skill and sub-skill criteria. Rather different from the studies mentioned previously, Saliu [5] has carried out a study using fuzzy inference system (FIS) approach based on Constrained Qualitative Assessment (CQA). Based on Saliu's work, the criteria are used as a guide to evaluate students in a computer system design course. 


\section{Fuzzy Inference System (FIS) and Monotonicity Property}

\subsection{Fuzzy Inference System}

Fuzzy inference system is a way to map an input space to an output space using fuzzy logic. There are four modules in developing FIS: a fuzzified input represented in a fuzzy set, often called fuzzification; pre-defined knowledge base storing IF-THEN rules; the inference engine to simulate human reasoning process using inputs and pre-defined rules; and defuzzification to transform obtained sets into a crisp value.

Assume a FIS input with $n$ inputs. Let's consider $x=\left(x_{1}, x_{2}, \ldots, x_{n}\right)$ as the input for the FIS. The inputs use linguistic terms of $A_{i}^{1}, A_{i}^{2}, \ldots, A_{i}^{L}$ which can be represented in a fuzzy membership function $\mu_{i}^{1}\left(x_{i}\right), \mu_{i}^{2}\left(x_{i}\right), \ldots, \mu_{i}^{L}\left(x_{i}\right)$, respectively. The pre-defined rule base is represented in an antecedent-consequent link in the form of IF-THEN rules such that:

$$
\text { IF } x_{1} \text { is } \tilde{A}_{1}^{l} \text { and } x_{2} \text { is } \tilde{A}_{2}^{l}, \ldots, x_{n} \text { is } \tilde{A}_{n}^{l} \text { THEN } y^{l} \text { is } \tilde{B}^{l} \text {; for } k=1,2 \ldots, n \text {. }
$$

where $\tilde{A}_{1}^{l}, \tilde{A}_{2}^{l}, \tilde{A}_{n}^{l}$ refers to fuzzy sets representing $l$ th antecedents pairs and $\tilde{B}^{l}$ is the fuzzy set representing the $l$ th consequent. The inference process is conducted to obtain the output using the combination survey data value, represented in fuzzy values, and predefined rules. The proposed system output is obtained using Mamdani implication given as:

$$
\mu_{\tilde{B}^{l}}(y)=\max _{l}\left[\min \left[\mu_{\tilde{A}_{1}^{l}}(\operatorname{input}(i)), \mu_{\tilde{A}_{2}^{l}}(\operatorname{input}(j))\right]\right], l=1,2, \ldots, r
$$

The result obtained from the implication is in the form of fuzzy sets. The sets are defuzzified to get the crisp value using Center of Gravity (COG) method.

\subsection{Monotonicity}

Assuring monotonic property in a real system such as an educational system is important. Some studies have highlighted the importance of the monotonicity in a fuzzy inference system [3][13][14]. A FIS that fulfills the condition of monotonicity between the outputs and its corresponding inputs satisfies the order given in Equation 3.

$$
f\left(x_{1}, x_{2}, \ldots, x_{i}^{1}, x_{n}\right) \leq f\left(x_{1}, x_{2}, \ldots, x_{i}^{2}, x_{n}\right) \text { as } x_{i}^{1}<x_{i}^{2}
$$

To satisfy the monotonic property, as the input increases, the output of FIS also increases monotonically. There are two conditions to satisfy monotonicity. The first condition states a method of tuning the membership function to ensure the developed FIS satisfies monotonicity. Let us assume both membership functions $\mu_{a}$ and $\mu_{b}$ are different. The condition that has to be fulfilled according to Koczy and Hirota [15] and Tay and Lim [3] is for $\mu_{a} \prec \mu_{b}$, extending Equation 4 to Equation 5 as follows:

$$
\begin{gathered}
\mu^{p^{\prime}}(x) \times \mu^{q}(x)-\mu^{p}(x) \times \mu^{q^{\prime}}(x) \leq 0 \\
\frac{\mu^{p^{\prime}}(x)}{\mu^{p}(x)} \leq \frac{\mu^{q^{\prime}}(x)}{\mu^{q}(x)}
\end{gathered}
$$


The second condition to satisfy the monotonicity property is to confirm a monotonic pre-defined rule base in the FIS. The second condition can be fulfilled by tuning the rules.

\section{Proposed FIS based on Affective and Cognitive Model}

\subsection{Cognitive and Affective Factors}

The cognitive factor used in this study is defined by scores obtained during the teaching and learning activities, which include tests, small tests, quizzes, and/or assignments. Commonly, the teaching and learning activities begin by determining objectives and expected outcomes, followed by instructional activities of the teaching and learning process. Tests, small tests, or quizzes are conducted to assess the student learning progress during the learning activities. The scores covering small tests, quizzes, and assignments and the final examination are aggregated to form a student cognitive score.

Affective factors on the other hand are, among other things, related to student motivation, attitudes, and feelings [8], specifically in English learning [16]. A method to quantify the student affective factors is proposed using the Likert scale with response values ranging from "strongly agree" to "strongly disagree" with a "neutral option' in the middle. The outcome of this method is a set of affective questionnaires intended to measure student affective level quantitatively.

The development of the affective questionnaire began with previous works in English learning [16][17][18][19]. Based on these works, affective factors that affect students in English language learning are determined to include motivation, introversion, extroversion, and anxiety. Further, indicators of the determined factors are identified as a basis to formulate the affective questionnaire. After being edited and reviewed by an English language education expert, a preliminary test is conducted to perform alpha analyses prior to survey data collection and to check the questionnaire consistency. Overall, the alpha values for each factor ranges from moderate to good alpha values of $.823, .531, .645, .838$ for motivation, introversion, extroversion, and anxiety, respectively.

\subsection{Proposed Fuzzy Inference System}

Figure 1 shows the overall step of the proposed cognitive affective assessment using fuzzy set theory. The steps are divided into two parts, first is the development of the cognitive assessment and the second is the development of the affective assessment. The development of the cognitive assessment is generally similar to the teaching and learning activities. First, learning objectives and learning outcome of the study are determined. Next, learning activities are conducted and students are then assessed based on learning objectives through learning activities such as assignments, quizzes, and exams. The result of the student assessment is the averaged score obtained of English skills (listening, speaking, reading, and writing) used as the foundation of the student cognitive criterion. Both factors are fuzzified after the cognitive criterion and affective criteria are collected.

\subsection{Fuzzification and Fuzzy Rules}

The process of fuzzification involves one cognitive factor and four affective factors. Each of the affective factors of the scoring criteria can be represented by a fuzzy set by using linguistic values. First, the linguistic terms employed for assigning the learning score of the 


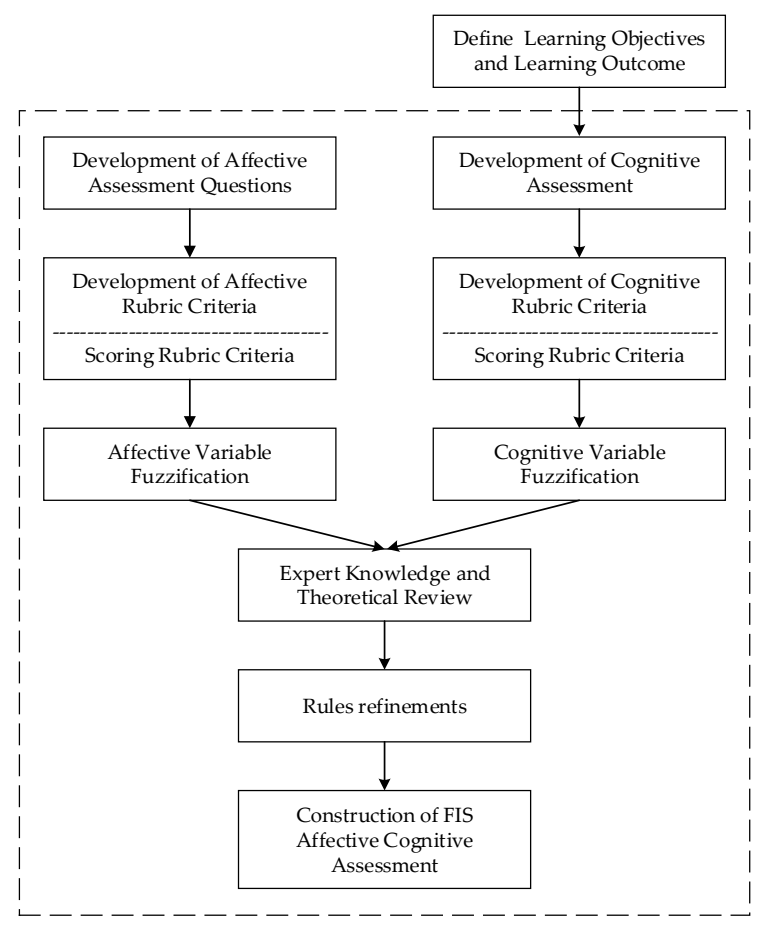

Figure 1: Proposed Affective-Cognitive FIS

cognitive factor are as follows: Elementary, Pre-Intermediate, Intermediate, Pre-Advance, and Advance with the value associated with them. The fuzzy set of learning score is denoted by $C_{S c}^{l}=\{E, P I, I, P A, A\}$. For example, a student with a score of 60 is assigned to 'Pre-Intermediate', which refers to learning-score criteria. Figure 2 illustrates the fuzzy membership functions of the cognitive factor $\left(\mu_{S c}^{l}\right)$.

Membership functions of affective factors are Motivation $\left(\mu_{M t}^{l}\right)$, Introversion $\left(\mu_{I t}^{l}\right)$, Extroversion $\left(\mu_{E x}^{l}\right)$, and Anxiety $\left(\mu_{A x}^{l}\right)$. The affective factor has the linguistic terms of Very Low, Low, Moderate, High, and Very High with values assigned to each of them. The set of the linguistic terms of $A_{j}^{l}=\{V L, L, M, H, V H\}$ applies to all of the affective factors where $j$ denotes the affective factors and $l$ denotes the membership value. For example, the fuzzy set of motivation is denoted by $A_{M t}^{l}=\{V L, L, M, H, V H\}$, as shown in Figure 2.

A student's final score is in a range from 0 to 100, represented by five qualitative verbal terms: Unsatisfactory, Fair, Good, Very Good, and Excellent. The corresponding value for each of the final score are 0 - 44 for unsatisfactory, 45 - 55 for fair, 56 - 69 for good, 70 80 for good, and 81 - 100 for excellent.

Fuzzy rules to infer student learning achievement are based on the theoretical framework synthesized from literature about English learning. The rules are then edited by to an English expert. The representation of fuzzy rules in this study can be symbolized by:

$$
\begin{aligned}
& \text { IF } x_{1} \text { is } \mu_{M t}^{l} \text { and } x_{2} \text { is } \mu_{I t}^{l} \text { and } x_{3} \text { is } \mu_{E x}^{l} \text { and } x_{4} \text { is } \mu_{A x}^{l} \text { and } x_{5} \text { is } \mu_{S c}^{l} \text { THEN } y \text { is } \mu_{F s}^{l} \\
& \quad \text { for } l=1,2 \ldots, n \text {. }
\end{aligned}
$$

An sample of fuzzy rules can be seen in Table 1 . 

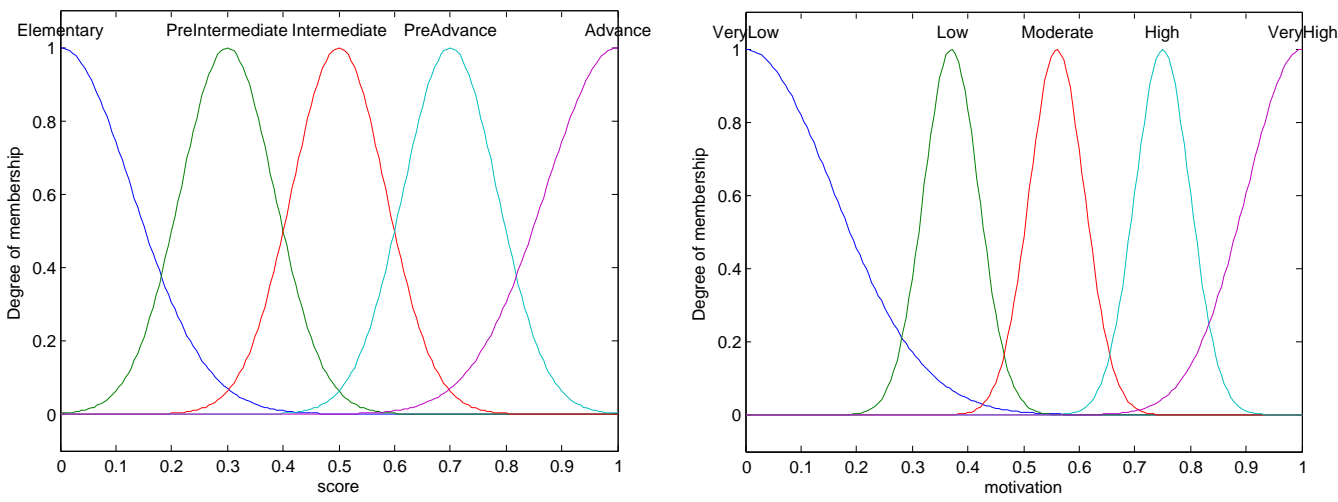

Figure 2: Example of membership functions

Table 1: An example of fuzzy rules

Fuzzy Rules

1 IF (Motivation is High) AND (Introversion is Low) AND (Extroversion is High) AND (Anxiety is Low) AND (Learning Score in Pre-Advance) THEN Achievement is Very Good [1]

2 IF (Motivation is Low) AND (Introversion is High) AND (Extroversion is Low) AND (Anxiety is High) AND (Learning Score in Pre-Intermediate) THEN Achievement is Fair [1]

\subsection{Fuzzy Rules Refinement through Monotonicity Property}

Monotonicity is an important property for creating a FIS to produce a valid and meaningful comparison among student achievements. The monotonic function describes the relationship between affective and cognitive input, and student achievements. Based on the theoretical review on affective factors [16][17][18], a student with a high learning score possesses a high level of motivation and extroversion and a low level of introversion and anxiety. Among the affective factors, motivation is the most influential factor, followed by extroversion, anxiety, and introversion, respectively [16]. Thus, a student with these attributes is expected to obtain a higher level of achievement. For example, the monotonicity suggests that the student with the highest level of motivation should have an achievement that is equal or higher than the other students.

Two conditions are applied to the FIS model to preserve monotonicity. Condition one is used as a guidance to develop the affective membership functions and the cognitive membership function. A derivation technique can be used to visualize the monotonicity that satisfies condition one [3]. Let's assume that the construction of the membership function is based on the Gaussian membership function. Deriving the function in Equation 7 and using Equation 5, it results in a linear function, as follows:

$$
\begin{gathered}
F(x)=e^{\frac{-[-x-c]^{2}}{2 \sigma^{2}}} \rightarrow F^{\prime}(x)=-\left(\frac{(x-c)}{\sigma^{2}}\right) F(x) \\
G(x)=\frac{G^{\prime}(x)}{\left.G^{(} x\right)}=-\left(\frac{1}{\sigma^{2}}\right) x+\left(\frac{c}{\sigma^{2}}\right)
\end{gathered}
$$


Calculating the membership function using Equation 8 shows $E_{\text {VeryHigh }}(x)>E_{H i g h}(x)>$ $E_{\text {Moderate }}(x)>E_{\text {Low }}(x)>E_{\text {VeryLow }}(x)$. To satisfy the condition two, adjustment of the predefined rules is performed.

\section{Results}

\subsection{Assessment Results}

Table 2 summarizes the example result of the assessment based on the FIS. As seen in Table 2 there are 2 test case data as represented in column "No". Each column of the affective score lists the student affective level of "Motivation", "Introversion", "Extroversion", and "Anxiety", while column for "Cognitive Scores" list the student learning score. The result of the assessment is shown in two types: fuzzy scores and description terms. The last column shows the rule assessment after the rule refinement.

Table 2: Results of assessment based on FIS

\begin{tabular}{|c|c|c|c|c|c|c|c|c|}
\hline \multirow{2}{*}{ No } & \multicolumn{4}{|c|}{ Affective Level } & \multirow{2}{*}{$\begin{array}{c}\text { Cognitive } \\
\text { Score } \\
\text { Sc }\end{array}$} & \multicolumn{2}{|c|}{ FIS Assessment } & \multirow{2}{*}{ Refined FIS } \\
\hline & Mt & It & Ex & Ax & & Fuzzy Score & $\begin{array}{l}\text { Linguistic } \\
\text { Terms }\end{array}$ & \\
\hline 1 & .67 & .36 & .73 & .44 & .90 & 85.82 & Excellent & 85.22 \\
\hline 2 & .88 & .36 & .73 & .44 & .90 & 90.14 & Excellent & 90.11 \\
\hline
\end{tabular}

Both students, students 1 and 2, have the same affective value of introversion (.36), extroversion (.73), anxiety (.44), and learning score (.90). However, student 1 has higher motivation. The monotonicity property suggests that a student with higher motivation should have higher achievement than a student with lower motivation. From the observations of test cases 1 and 2, the modeled FIS is able to satisfy the monotonicity property.

Surface plots are shown to illustrate the proposed FIS. The inputs were reduced to a lower dimensionality by pairing two inputs to visualize the mapping of the system. The input pairs are the affective factor and the cognitive factor. The example of mapping infers student achievement as shown in Figure 3. The mapping sets the two factors in a fixed value and other factors span the surface with a value ranging from 0 to 1 . The mapping shows non-linearity with some slopes and the surface is monotonic.

\subsection{Simulation}

This study uses 188 sets of previous student surveys data [20] and 188 sets of pseudorandomly generated data to investigate the properties of the FIS in use. The result shown in Table 3 indicates that input factors embracing motivation, introversion, extroversion, anxiety, and student learning score are significantly correlated. The affective factors of motivation and extroversion have a positive correlation with students final score, while introversion and anxiety have a negative correlation. The cognitive factor, indicated by student learning scores, is correlated positively with student final score. The correlation result supports previous studies [16][17][18][19] by showing a positive correlation of the positive affect: 


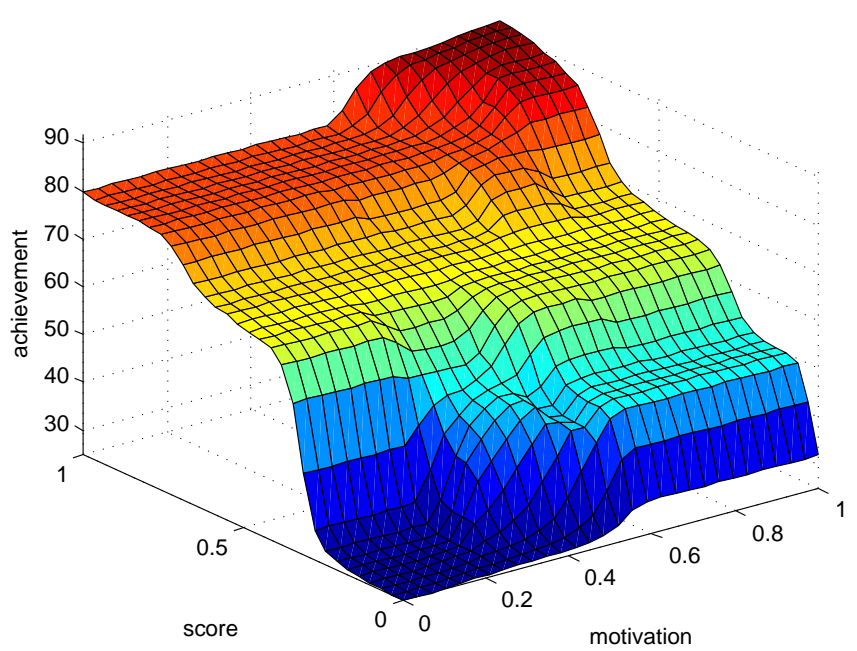

Figure 3: Achievement surf plot

Table 3: Survey data simulation

\begin{tabular}{llllll}
\hline & Mt & It & Ex & Ax & Sc \\
\hline \hline Correlation & $.526^{* *}$ & $-.145^{*}$ & $.677^{* *}$ & $-.304^{* *}$ & $.603^{* *}$ \\
Sig. (2-tailed) & .001 & .047 & .001 & .001 & .001 \\
\hline${ }^{* *} p<.01{ }^{*} p<.05$ & & & &
\end{tabular}

Table 4: Pseudo-random data simulation

\begin{tabular}{lccccc}
\hline & Mt & It & Ex & Ax & Sc \\
\hline \hline Correlation & .088 & -.117 & .005 & -.052 & $.170^{*}$ \\
Sig. (2-tailed) & .229 & .111 & .945 & .477 & .020 \\
\hline${ }^{* *} p<.01 ;{ }^{*} p<.05$ & & & &
\end{tabular}

motivation and extroversion, and a negative correlation of the negative affect: introversion and anxiety with student achievement.

The result of survey data simulation and pseudo-random data generation is different. As seen in Table 4, all of the affective factors, motivation, introversion, extroversion, and anxiety, are not significantly correlated with student achievement. However, only the cognitive factor is correlated with student achievement $\left(.170^{*}, p<.05\right)$. The results shown Table 4 , indicate cases of correlation between student affective and cognitive values do not occur in a significant number of cases in random simulations.

\section{Conclusion}

This paper has presented a result of establishing affective-cognitive FIS - a method of assessing student achievement in English learning by considering affective factors and cogni- 
tive factors. The process of building the system includes confirming the property of monotonicity, crucial to ensure the system validity. The monotonicity property was applied in the development of the membership functions and rule refinement. In the current work the student affective level is measured using a set of questionnaires developed specifically to measure student affective attributes. The result of this study shows a basic method utilizing both affective and cognitive factors that might be used in real practice. The proposed system is able to produce a sufficient output that is expected to be able to support teachers in the assessment processes.

The factors that influence achievement need to be adjusted in order to develop questionnaires for subjects other than English language. In addition, a method to select a pre-defined rule base is needed by starting with a small set of rules. A sequential step to add the rule to the inference system is preferred. A specific method could be applied to create a robust pre-defined rule. Two of the techniques are rule interpolation [21][22] optimizing selected rules [23] and others.

\section{References}

[1] J. Salvia and J.E. Ysseldyke, "Assessment" (8th edition). Boston: Houghton Mifflin Company, 2001.

[2] F. M. Cin and A. F. Baba, "Assessment of English proficiency by fuzzy logic approach," International Educational Technology Conference, 2008, pp. 355-359.

[3] K. M. Tay and C. P. Lim, "A fuzzy inference system-based criterion-referenced assessment model," Expert System with Applications, Elsevier, vol.38, no. 9, 2011, pp. 11129-11136.

[4] D. R. Sadler, "Interpretations of criteria-based assessment and grading in higher education," Assessment \& Evaluation in Higher Education, Taylor \& Francis, vol. 30, no.2, 2005, pp. 175-194.

[5] S. Saliu, "Constrained subjective assessment of student learning," Journal of Science Education and Technology, Springer, vol 14, no. 3, 2005, pp 271-284.

[6] J. Biggs, "Teaching for quality learning at university," Society for Research into Higher Education and Open University, 1999.

[7] M. H. Immordino-Yang and A. Damasio, "We feel, therefore we learn: The relevance of affective and social neuroscience to education," Mind, brain, and education, Wiley Online Library, vol. 1, no. 1, 2007, pp. 3-10.

[8] K. Shephard, "Higher education for sustainability: seeking affective learning outcomes," International Journal of Sustainability in Higher Education, Emerald Group Publishing Limited, vol. 9, no. 1, 2008, pp. 87-98.

[9] L. W. Anderson, et al., "A taxonomy for learning, teaching, and assessing: A revision of Bloom's taxonomy of educational objectives, abridged edition," White Plains, NY:Longman, 2001.

[10] R. Biswas, "An application of fuzzy sets in students' evaluation," Fuzzy sets and systems, Elsevier, vol. 74, no. 2, 1995, pp. 187-194. 
[11] S. M. Chen and C. H. Lee, "New methods for students' evaluation using fuzzy sets," Fuzzy sets and systems, Elsevier, vol. 104, no. 2, 1999, pp. 209-218.

[12] S. M. Bai, S. M. Chen, "Automatically constructing grade membership functions of fuzzy rules for students evaluation," Expert Systems with Applications, Elsevier, vol. 35, no. 3, 2008, pp. 1408-1414.

[13] H. Seki, H. Ishii, and M. Mizumoto, "On the monotonicity of fuzzy-inference methods related to T-S inference method," Fuzzy Systems, IEEE Transactions on, IEEE, vol. 18, no. 3, 2010, pp. 629-634.

[14] H. Zhao and C. Zhu, "Monotone fuzzy control method and its control performance," Systems, Man, and Cybernetics, 2000 IEEE International Conference on, IEEE, pp. 3740-3745, 2000.

[15] L. Kóczy and K. Hirota, "Ordering, distance and closeness of fuzzy sets," Fuzzy Sets and Systems, Elsevier, vol. 59, no. 3, 1993, pp. 281-293.

[16] H. D. Brown, "Affective variables in second language acquisition," Language learning, Wiley Online Library, vol. 23, no. 2, 1973, pp. 231-244.

[17] A. Al-Tamimi, M. Shuib, "Motivation and attitudes towards learning English: A study of petroleum engineering undergraduates at Hadhramout University of Sciences and Technology," GEMA Online Journal of Language Studies, vol. 9, no. 2, 2009, pp. 2955 .

[18] S. Zafar and K. Meenakshi, "A study on the relationship between extroversionintroversion and risk-taking in the context of second language acquisition," International Journal of Research Studies in Language Learning, vol. 1, no. 1, 2011.

[19] E. K. Horwitz, M. B. Horwitz, and J. Cope, "Foreign language classroom anxiety," The Modern language journal, Wiley Online Library, vol. 70, no. 2, 1986, pp. 125-132.

[20] F.A. Bachtiar, K. Kamei, E. W. Cooper, "A Neural Network Model of Students' English Abilities Based on Their Affective Factors in Learning," Journal of Advanced Computational Intelligence and Intelligent Informatics (JACIII), vol. 16, no. 3, 2012, pp. 375-380.

[21] L. T. Koczy, K. Hirota, "Size reduction by interpolation in fuzzy rule bases," Systems, Man, and Cybernetics, Part B: Cybernetics, IEEE Transactions on, IEEE, vol. 27, no. 1, 1997, pp. 14-25.

[22] R. Diao, S. Jin, and Q. Shen, "Antecedent selection in fuzzy rule interpolation using feature selection techniques," Fuzzy Systems (FUZZ-IEEE), 2014 IEEE International Conference on, IEEE, 2014, pp. 2206-2213.

[23] H. Ishibuchi, T. Yamamoto, "Fuzzy rule selection by multi-objective genetic local search algorithms and rule evaluation measures in data mining," Fuzzy Sets and Systems, Elsevier, vol 141, no. 1, 2004, pp. 59-88. 